\title{
Spatiotemporal Dynamics of Complement C5a Production within Bacterial Extracellular Polymeric Substance
}

\author{
Erin C. Conrad ${ }^{a} \quad$ Yueh-Ya Hsu $^{d} \quad$ David M. Bortz ${ }^{d} \quad$ John G. Younger ${ }^{a-c}$ \\ ${ }^{a}$ Department of Emergency Medicine, ${ }^{b}$ Michigan Center for Integrative Research in Critical Care, and \\ 'Biointerfaces Institute, University of Michigan, Ann Arbor, Mich., and d Department of Applied Mathematics, \\ University of Colorado, Boulder, Colo., USA
}

\section{Key Words \\ Complement $\cdot$ Computational modeling $\cdot$ Extracellular polymeric substance $\cdot$ Sepsis $\cdot$ Staphylococcus epidermidis}

\begin{abstract}
Opsonization and anaphylatoxin production are early events in the innate response to bacterial pathogens. Opsonization alone is frequently not lethal and production of anaphylatoxins, especially $\mathrm{C} 5 \mathrm{a}$, allows for recruitment of cellular defenses. Complement biochemistry is extensively studied and computational models have been reported previously. However, a critical feature of complement-mediated attack is its spatial dependence: diffusion of mediators into and away from a bacterium is central to understanding C5a generation. Spatial dependence is especially important in biofilms, where diffusion limitation is crucial to bacterial counterdefense. Here we develop a model of opsonization and C5a production in the presence of a common blood-borne pathogen, Staphylococcus epidermidis. Our results indicate that when complement attacks a single cell, diffusion into the extracellular polymeric substance (EPS) is complete within $10 \mathrm{~ms}$ and that production of C5a peaks over the next $15 \mathrm{~min}$. When longer diffusion lengths (as in an EPS-rich biofilm) are incorporated, diffusion limitation appears such that
\end{abstract}

the intensity and duration of C5a production is increased. However, the amount of $\mathrm{C} 5$ a produced under several likely clinical scenarios where single cells or sparse biofilms are present is below the $k_{D}$ of the $C 5$ a receptor suggesting that complement activation by a single bacterium may be difficult to detect when diffusion is taken into account.

Copyright $\odot 2013$ S. Karger AG, Basel

\section{Introduction}

The complement cascade is a complex network of soluble extracellular enzymes, protein substrates, membrane-bound receptors, and regulatory proteins responsible for detecting, marking for phagocytosis, and sometimes killing invasive microorganisms. Counter defenses against the opsonic and directly bactericidal functions of the complement cascade are common among successful pathogens; resistance to these strategies, particularly among Gram-positive bacteria, is the rule rather than the exception. Fortunately, in the course of its activation, the pathway also produces several extracellular signaling molecules, the anaphylatoxins $\mathrm{C} 3 \mathrm{a}, \mathrm{C} 4 \mathrm{a}$, and C5a. Acting through cell surface receptors, these signaling molecules induce potent host-defensive responses by innate and

\section{KARGER \\ Fax +4161306 1234 \\ E-Mail karger@karger.ch}

www.karger.com
(C) 2013 S. Karger AG, Basel

1662-811X/13/0052-0114\$38.00/0

Accessible online at: www.karger.com/jin
Dr. John G. Younger

Department of Emergency Medicine, University of Michigan TC B1 354

Ann Arbor, MI 48109-5303 (USA)

E-Mail jyounger@umich.edu 
adaptive immune cell types, vascular smooth muscle and endothelium, which together produce the local inflammatory response necessary to control infectious challenges [1]. Thus, the anaphylatoxins play a particularly important role as a means of attracting and coordinating cellular responses to a location where the humoral arsenal of complements is likely to fail [2].

As with many pro-inflammatory host strategies, there are ramifications to uncontrolled complement activation, particularly related to unfettered production of C5a. When C5a is produced in sufficient quantities to act systemically, rather than at a site of infection, it leads to low blood pressure, widespread increases in vascular permeability, cardiac dysfunction, and apoptosis of lymphocytes [3-7]. In animal models of sepsis, the most extreme example of uncontrolled host response to infection, C5a, is an important contributor to mortality; blockade by anti-C5a antibodies or deletion of $\mathrm{C} 5 \mathrm{a}$ receptors is significantly protective [8-10]. Our laboratory recently demonstrated significant elevation of $\mathrm{C} 3 \mathrm{a}$ and $\mathrm{C} 5 \mathrm{a}$ in the blood of severely septic patients at the time of initial presentation to the emergency department [11].

The abundance of complement proteins in plasma, the availability of quantitative functional assays, and the straightforward architecture of the enzyme network have allowed for detailed descriptions of the enzyme kinetics of this system [12-14]. Such meticulous effort opens the door for mathematical modeling of complement behavior, and some work has been reported [15-19]. The value of such work lies in the ability to comment on important phenomena that occur at short distances and over brief times that, while relevant to individual cells, are inaccessible to existing experimental techniques. One feature that has not been incorporated into previous work is the inherently spatial nature of complement activation. While capable of activating in solution, complement activation primarily occurs on the surface of a detected threat. The presence of a surface requires consideration of diffusion and convection to and away from the site of activation and suggests that flux (change in concentration per unit surface area) is a natural measure of complement activity in the setting of host defense. Thus, in general this approach provides an opportunity to consider the complex chemistry of the complement cascade over time in three-dimensional space.

In the current work, we pursue this idea in the context of a common life-threatening condition, namely blood stream infection with Staphylococcus epidermidis. S. epidermidis is a ubiquitous skin-dwelling bacterium that is the most frequent cause of indwelling medical device-as- sociated infections and typically ranks in the top 3 or 4 most frequently identified causes of bacteremia [20, 21]. Unlike many bacteria of medical interest, S. epidermidis is not typically armed with an array of pathogenic factors. Rather, its ability to cause disease is primarily a function of its complex extracellular network of polysaccharides, proteins, and, in the case of biofilms, nucleic acids, collectively known as extracellular polymeric substance (EPS). This structure promotes biofilm formation and defends against complement-mediated attack and phagocytosis while nevertheless serving as an effective complement activator [22]. Thus, S. epidermidis is an excellent species with which to explore spatiotemporal complement behavior from a computational perspective.

Here, we consider what may be the most important issue in understanding complement pathway interactions with S. epidermidis, namely how does the presence of a thick layer of surface material influence the kinetics of complement activation against this pathogen? We begin by developing and analyzing a mathematical description of complement diffusion, binding, and catalysis within the EPS surrounding a single bacterium. This model is used to establish the timing and intensity of $\mathrm{C} 5$ a production near the surface of a complement-resistant bacterium and to predict the effectiveness of C5a-mediated signaling to nearby neutrophils or endothelial cells as would be expected in the bloodstream. Lastly, we evaluate the impact of $S$. epidermidis growing in its most common disease-associated state - a surface-adherent biofilm and assess the importance of mediator diffusion limitation in the dynamics of $\mathrm{C} 5$ a production in biofilms.

\section{Materials and Methods}

Sources of Rate Constants and Corroborative Experimental

Data

When possible, enzymatic rate constants and C3, C5, and C5a concentrations were taken directly from the literature and are shown in table 1. EPS thickness was taken from two published methods, including dynamic light scattering of suspended cells and high-resolution spatial analysis of $S$. epidermidis growing in biofilms [23, 24]. Diffusion coefficients for complement proteins in biofilm were not available. These values were therefore estimated from fluorescence recovery after photobleaching diffusion measurements of high-molecular-weight dextrans with comparable hydrodynamic radii to the complement proteins under consideration [25]. Computational results were compared to multiple published sets of experimental observations. Timing of C3 opsonization was compared first to similar experimental work with Klebsiella pneumoniae [19]. Raw flow-cytometric data files from that manuscript were re-analyzed to provide the fraction of all counted cells in which any amount of C3 binding was detected. 
Table 1. Model parameters and values

Geometry and initial conditions

Radius, cell center to cell edge

$0.22 \mu \mathrm{m}[24]$

Radius, cell center to EPS edge distance

$0.50 \mu \mathrm{m}[24]$

Serum C3 concentration

$1.6 \times 10^{3}$ molecules/ $\mu \mathrm{m}^{3}[26]$

Initial EPS concentration

$3.3 \times 10^{4}$ binding sites $/ \mu \mathrm{m}^{3}[26,27]$

Serum C5 concentration

$2.2 \times 10^{2}$ molecules/ $\mu \mathrm{m}^{3}[34]$

Serum C5a concentration

$4.7 \times 10^{-2}$ molecules $/ \mu \mathrm{m}^{3}[35]$

\begin{tabular}{ll} 
Diffusivity and rate constants & \\
$D_{C 3}$, C3 diffusion coefficient & $19 \mu \mathrm{m}^{2} / \mathrm{s}[25]$ \\
$D_{C 5}$, C5 diffusion coefficient & $18 \mu \mathrm{m}^{2} / \mathrm{s}[25]$ \\
$D_{C 5 a}$, C5a diffusion coefficient & $85 \mu \mathrm{m}^{2} / \mathrm{s}[25]$ \\
$K_{C 3}$, activated C3 binding rate constant & $4.2 \times 10^{-10}(\mathrm{~m})$ \\
$K_{\text {tickover }}$, spontaneous C3 binding rate constant & $1.0 \times 10^{-6} \cdot \mathrm{s}^{-1}[13$ \\
$K_{\text {decayy }}$, decay rate of C3/C5 convertase & $8.0 \times 10^{-3} \cdot \mathrm{s}^{-1}[36$ \\
$K_{C 5}$, C5 convertase rate constant & $6.0 \times 10^{-6}(\mathrm{mo}$ \\
\hline Vessel and neutrophil geometry & \\
Capillary radius & $5 \mu \mathrm{m}$ \\
$v$, mean capillary plasma velocity & $0.5 \mathrm{~mm} / \mathrm{s}[29]$ \\
Neutrophil radius & $5 \mu \mathrm{m}$
\end{tabular}

Timing was also compared to an ELISA-based strategy for quantifying C3 binding to encapsulated strains of Cryptococcus neoformans $[26,27]$. C5a flux was compared to measurements taken from $S$. epidermidis biofilms grown on plastic pins and submersed in human serum [28]. In that work, biofilms were grown on plastic pegs (cylinders with a length of $1.25 \mathrm{~mm}$ and a radius of 1.75 $\mathrm{mm}$ ), with a hemispherical end cap, providing a total biofilm surface area of $3.3 \times 10^{7} \mu \mathrm{m}^{2}$. To compare our computational prediction of $\mathrm{C} 5$ a production to the values noted in that report, we assumed a $1-\mu \mathrm{m}$-thick biofilm completely covering the available peg surface area and exposed to serum for $30 \mathrm{~min}$.

\section{Mathematical Specification of Complement Reactions}

Modeled reactions are shown in figure 1a. We considered only 6 species: free C3, free EPS (simplified schematically here as a polysaccharide but in reality any surface-associated extracellular structure), C3/C5 convertase bound to EPS, inactive bound C3/ C5 convertase, free C5, and free C5a. The model is stated formally in the online supplementary material (for all online suppl. material, see www.karger.com/doi/10.1159/000345705). Most mathematical analysis was performed in a spherically symmetric model of a single bacterium (fig. 1b). The most important spatial domain of this model is the EPS, conceptualized as a uniform hydrogel available for complement protein diffusion and reaction, bound on its inner edge by a complement-impermeable cell wall and on its outer edge by the EPS-plasma interface. Neither the plasma surrounding the bacterium nor species diffusing and reacting through the EPS were considered well mixed, such that spatial gradients could develop over time. Subsequent modeling considered a single bacterium adjacent to the wall of a capillary with a radius of $5 \mu \mathrm{m}$ through which acellular plasma flowed at $0.5 \mathrm{~mm} / \mathrm{s}$ [29]. The reaction-diffusion equations were modified to include convection using the advective-diffusion equation [30]. In this model, C3, C5, and C5a concentrations were held at plasma levels at the inlet of the capillary. The endothelial wall was assumed to be a non-absorbing insulated boundary. The third geometry considered was a single bacterium in close proximity to a neutrophil of $5 \mu \mathrm{m}$ radius.

\section{Initial and Boundary Conditions}

$t_{0}$ was defined as the moment of first contact between bacteria and host plasma. At $t_{0}$, no complement proteins were within the EPS envelope and all EPS was available for opsonization. Concentrations of C3, C5, and C5a at the free EPS edge at $t_{0}$ were assumed to be normal plasma concentrations, as shown in table 1 . At subsequent times, plasma concentrations were held constant (i.e., served as a continuous source) at a distance, from the free edge, of ten times the total EPS thickness. The bacterial cell edge was assumed impermeable to complement proteins and was treated as an insulated boundary.

\section{Numerical Methods}

MATLAB (MathWorks Inc., Natick, Mass., USA) was used to solve the model equations for the single bacterium and the planktonic solution. It was also used to perform the simplex optimization algorithm (with the function fminsearch) used to determine the values for the rate constant of C3 binding to EPS and the initial EPS concentration from published data described above. COMSOL Multiphysics (COMSOL Inc., Burlington, Mass., USA) was used to verify the MATLAB solution of the single bacterium and to solve the capillary model and neutrophil models.

Sensitivity and Stability Analysis

A differential sensitivity analysis was performed to determine which model parameters most strongly influenced the amount or timing of C5a production. A stability analysis was also performed 
Fig. 1. Species, reactions, and geometries considered. a Reaction scheme for C5a production from complement interactions with bacterial EPS. I. Initial spontaneous binding of $\mathrm{C} 3$ to an EPS binding site. Upon binding, the C3-EPS complex becomes the C3/C5 convertase for subsequent reactions. Note: throughout this figure, we depict binding to a polysaccharide, although in reality several protein and DNA targets likely exist. None of the modeling is affected by this point. II. C3 convertase activation of additional C3. III. C5 convertase activation of $\mathrm{C} 5$, releasing diffusible C5a into the environment. IV. Spontaneous deactivation of the C3/C5 convertase into an inactive state. See online supplementary materials for associated partial differential equations used to model these behaviors. b Model geometries used in this paper. From left to right: a spherically symmetric single cell covered with bacterial EPS of thickness $r$; a single cell adjacent to the wall of a capillary as might be expected during filtration during bacteremia, and a single bacterium adjacent to a host neutrophil. a
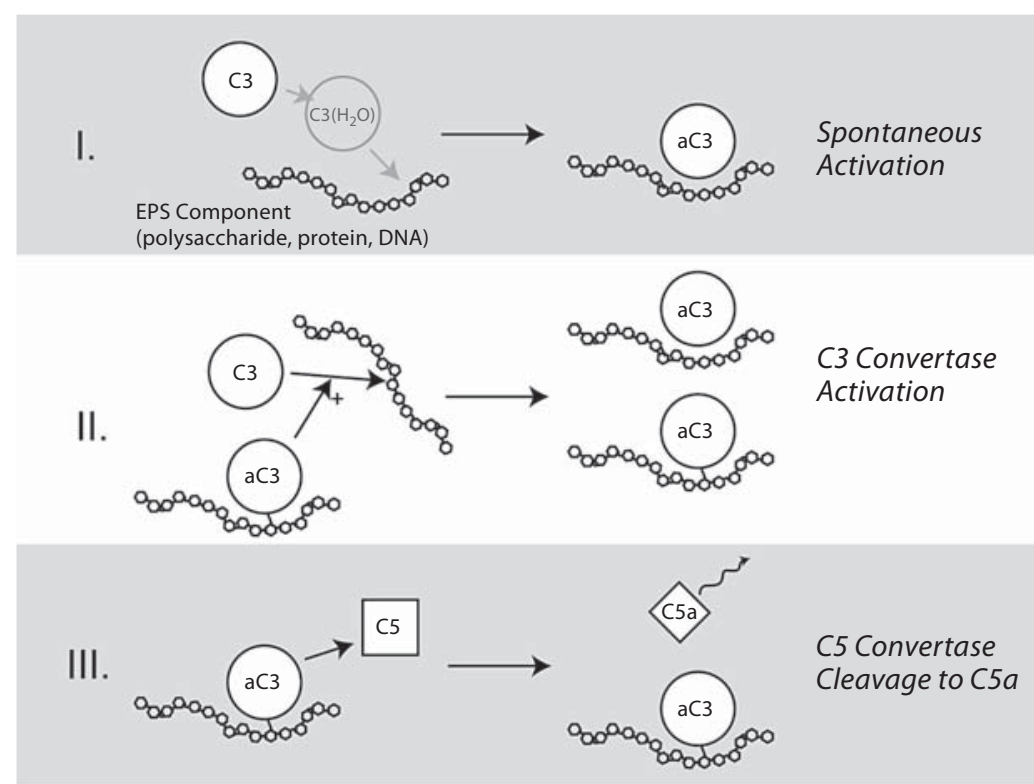

IV.
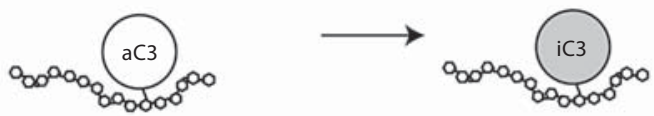

Spontaneous Deactivation b

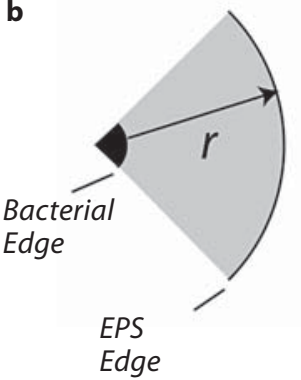

Edge
Endothelial-bound Neutrophil

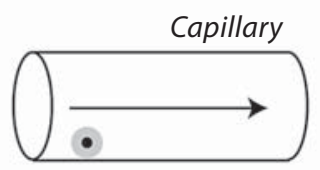
Bacterium
Adjacent

Bacterium

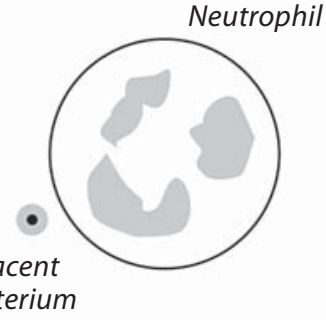

to examine the stability of the solution at equilibrium, using the standard method described by Murray [31]. The stability analysis revealed no instabilities in either the spatial or nonspatial model at equilibrium.

\section{Results}

The dynamics of $\mathrm{C} 3$ diffusion and C3/C5 convertase assembly into the EPS of a single bacterium are shown in figure 2. Using estimated diffusion coefficients derived from high-molecular-weight dextran movement through bacterial biofilms suggests that C3 movement into the
EPS is very rapid, reaching equilibrium with the plasma in approximately $10 \mathrm{~ms}$ (fig. 2a). The time course of opsonization was much slower, with peak $\mathrm{C} 3 / \mathrm{C} 5$ convertase activity (fig. 2b) occurring by $15 \mathrm{~min}$ of exposure to plasma, coincident with an approximately $50 \%$ reduction in EPS available for opsonization (fig. 2c). Also plotted in figure $2 \mathrm{c}$ are previously published data from our group using FITC-anti-C3 flow cytometry, and Kozel et al. [26, 27] and Nypaver et al. [19] using an ELISA to C3 to establish the opsonic time course for encapsulated microorganisms. These indicate that our spatial reaction-diffusion model, which was based solely on published enzymatic rate constants, complement protein concentrations, 


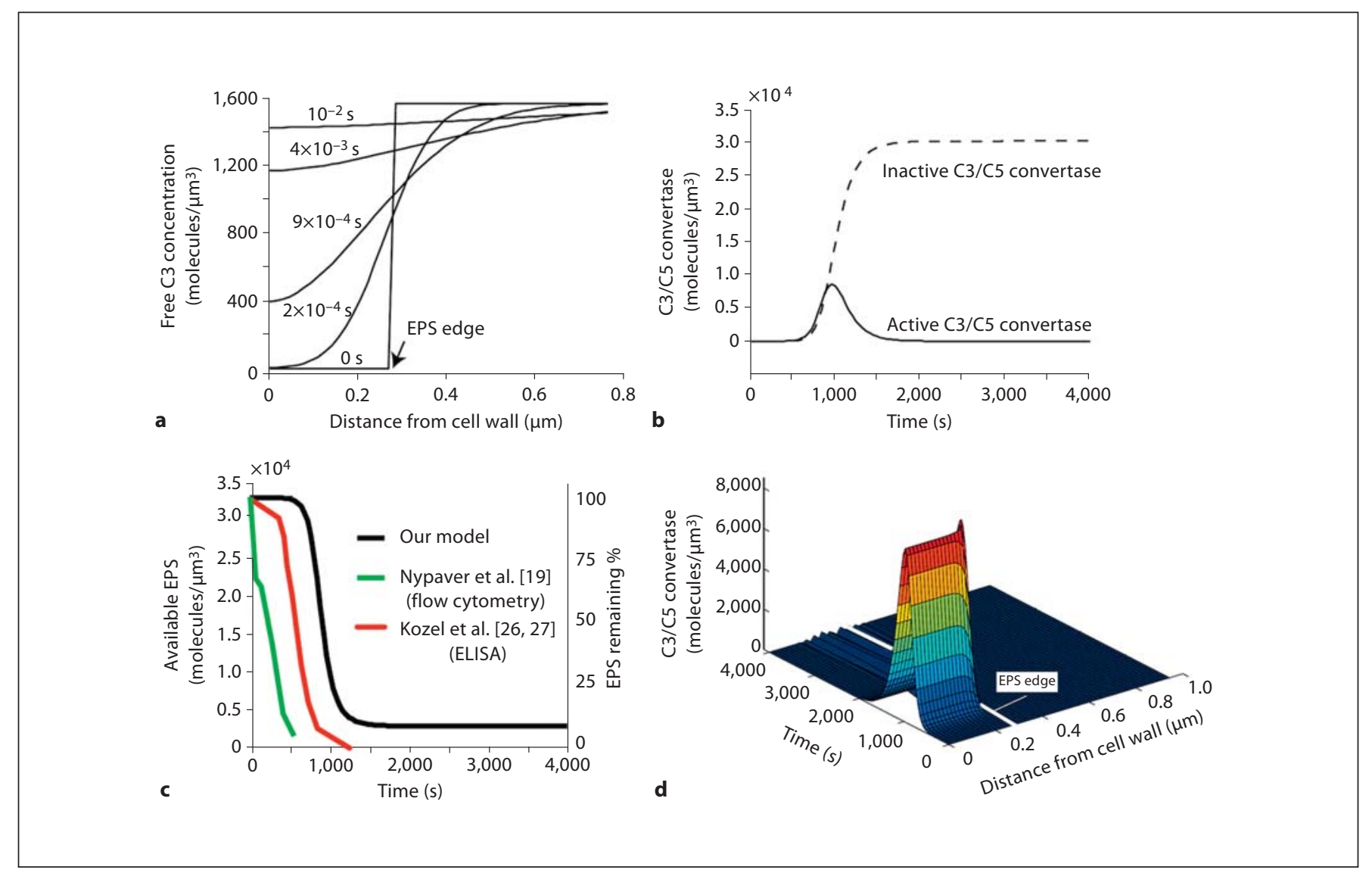

Fig. 2. Dynamics of C3 opsonization of a single EPS-bearing cell of $S$. epidermidis is not diffusion limited. a Rate of C3 diffusion into bacterial EPS. At $t_{0}$, no complement proteins are present internal to the EPS edge. Diffusion into the EPS is very fast, with near complete equilibrium between plasma levels and EPS levels achieved in $10 \mathrm{~ms}$. b Rate of C3 binding onto EPS. Binding of C3 to EPS, producing $\mathrm{C} 3 / \mathrm{C} 5$ convertase, takes place much more slowly than diffusion, with peak C3/C5 convertase activity present approximately $1,000 \mathrm{~s}$ into bacterial contact with the host. Within

and bacterial physical dimensions, produced estimates of the opsonization time course well within an order of magnitude of published independent experiments.

Ultimately, C3/C5 convertase activity within the EPS is lost due to degradation of enzymatic activity and almost complete depletion of available unbound EPS. Figure $2 \mathrm{~d}$ summarizes all of these results and predicts the essential dynamic behavior of opsonization of a single cell, specifically a rapid diffusive penetration throughout the EPS by $\mathrm{C} 3$ followed by a prolonged enzymatic interaction between the EPS and complement proteins. The inclusion of spatial diffusion into the model significantly changed the predicted behavior of the host-pathogen in-
$4,000 \mathrm{~s}, \mathrm{C} 3 / \mathrm{C} 5$ convertase activity ceased due to exhaustion of unbound EPS binding sites and spontaneous decay of active complexes. c Available binding sites for opsonization as a function of time. Also plotted are two experimental results taken from the literature, one examining K. pneumoniae opsonization via FITCanti-C3 flow cytometry and the other using an ELISA-based method to determine the time course of opsonization of $C$. neoformans. See text for details. d Spatiotemporal pattern of C3/C5 convertase activation.

teraction - when diffusion terms were left out of the model, the amount of active C3/C5 convertase produced was underestimated almost 20 -fold (online suppl. figure).

In the context of a single cell, C5 diffusion into and C5a diffusion out of the EPS is sufficiently rapid that any changes in C5a at the plasma-facing edge of the EPS and beyond are a function entirely of C3/C5 convertase activity throughout the EPS (fig. 3a). We performed a differential sensitivity analysis on the model to further evaluate the features that most determine the amount of $\mathrm{C} 5 \mathrm{a}$ elicited from the surface of a cell in contact with the host (fig. 3b). The diffusion coefficients for $\mathrm{C} 3$ and $\mathrm{C} 5$, which might be modulated by the pathogen by varying the den- 


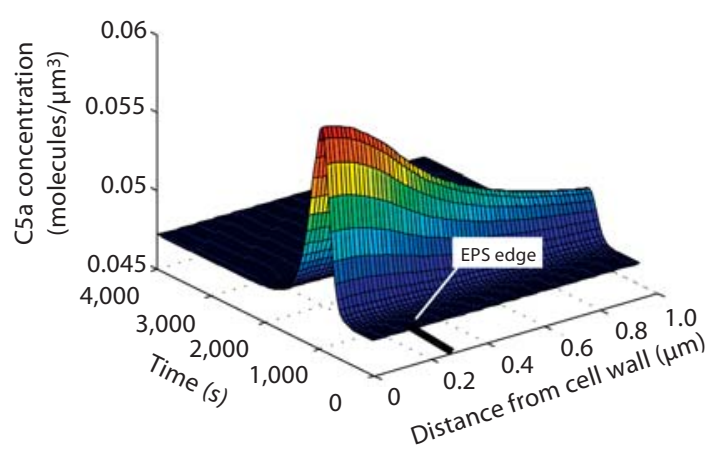

a

Fig. 3. C5a production during opsonization. a C5a concentration as a function of the distance from bacterial surface over time. The upstroke in $\mathrm{C} 5$ a production parallels the formation of active $\mathrm{C} 3$ / C5 complexes (fig. 2). The diffusivity of C5a is sufficiently fast that no meaningful gradient exists between the EPS layer and the surrounding plasma. C5a levels return to baseline within $4,000 \mathrm{~s}$ as a result of complete decay of $\mathrm{C} 3 / \mathrm{C} 5$ convertase activity and diffusion into the environment. Note the scale on the vertical axis: at

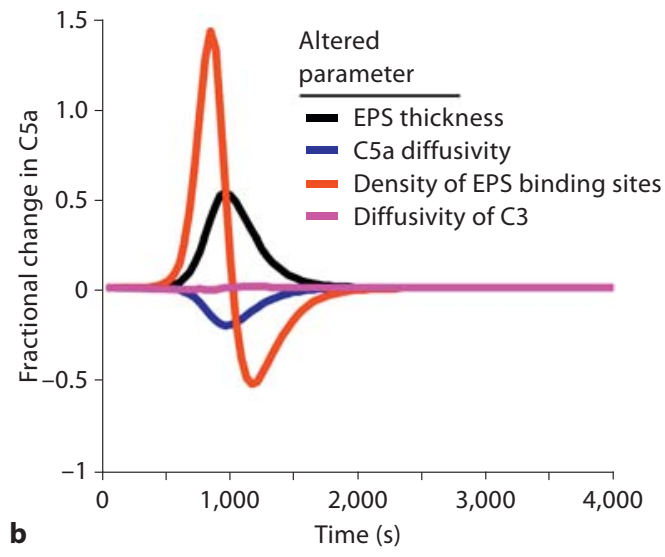

peak C5a production, the amount of C5a present is only $\sim 20 \%$ greater than background circulating levels reported in normal patients. b Sensitivity of C5a estimates to various model features. Note that after the initial reaction period the concentration of C5a in the EPS is unaffected by any of the parameters studied. During the reaction period, the initial concentration of EPS binding sites has the largest effect on the C5a concentration in EPS.

terium in close proximity and at the peak of its opsonization-related C5a release, a host cell would receive only a subtle perturbation in C5a-mediated signaling cascades. In fact, our model, on the basis of C5a alone, would predict that a single cell of $S$. epidermidis undergoing active opsonization could pass undetected in very close proximity to a host cell.

Lastly, as the primary pathogenic form of S. epidermidis is as a multicellular biofilm infecting an implanted medical device, we examined the effect of adding substantially more EPS thickness to the system. These results (fig. 6) indicate that when the envelope of extracellular material thickness exceeds $10 \mu \mathrm{m}, \mathrm{C} 3 / \mathrm{C} 5$ convertase behavior begins to demonstrate diffusion limitation, where the quantity of active enzyme complexes deep in the EPS gel is impeded by the time required for C3 to diffusively penetrate the material. For biofilms 50 and $100 \mu \mathrm{m}$ thick, this resulted in $\mathrm{C} 3 / \mathrm{C} 5$ convertase activity that was bimodal in time and sustained longer than 5,000 s. This sustained convertase activity was associated with increased and prolonged C5a production. In the case of a $100-\mu \mathrm{m}$ biofilm, C5a production persisted ten times longer than when activation occurred on the surface of a single cell. 


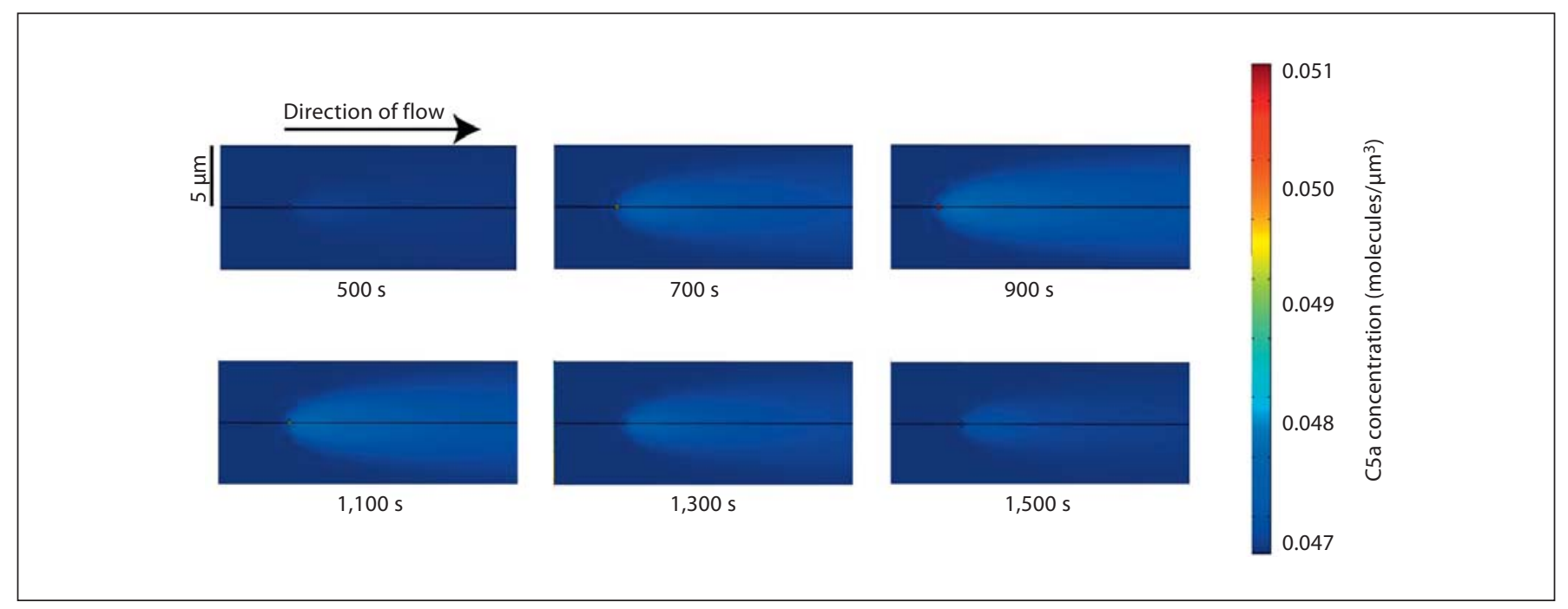

Fig. 4. C5a production from a bacterium trapped on a capillary wall. Using the geometry shown in figure $1 \mathrm{~b}$ and assuming continuous mean plasma velocity of $0.5 \mathrm{~mm} / \mathrm{s}$, the downstream plume of C5a from the surface of a single cell of S. epidermidis was modeled over $1,500 \mathrm{~s}$. The modest elevation in C5a produced by opsonization (fig. 3) in the presence of continuous dilution flow renders the change in concentration of $\mathrm{C} 5 \mathrm{a}$ on the endothelial surface very subtle - less than a $10 \%$ increase from plasma basal concentration. Of note, the $\mathrm{k}_{\mathrm{D}}$ for $\mathrm{C} 5 \mathrm{a}-\mathrm{C} 5 \mathrm{a}$ receptor binding is approximately $1 \mathrm{nM}\left(0.6\right.$ molecules/ $\left.\mu \mathrm{m}^{3}\right)$, thus even at peak $\mathrm{C} 5 \mathrm{a}$ production, the effective concentrations at the capillary wall are less than $10 \%$ of $\mathrm{k}_{\mathrm{D}}$.

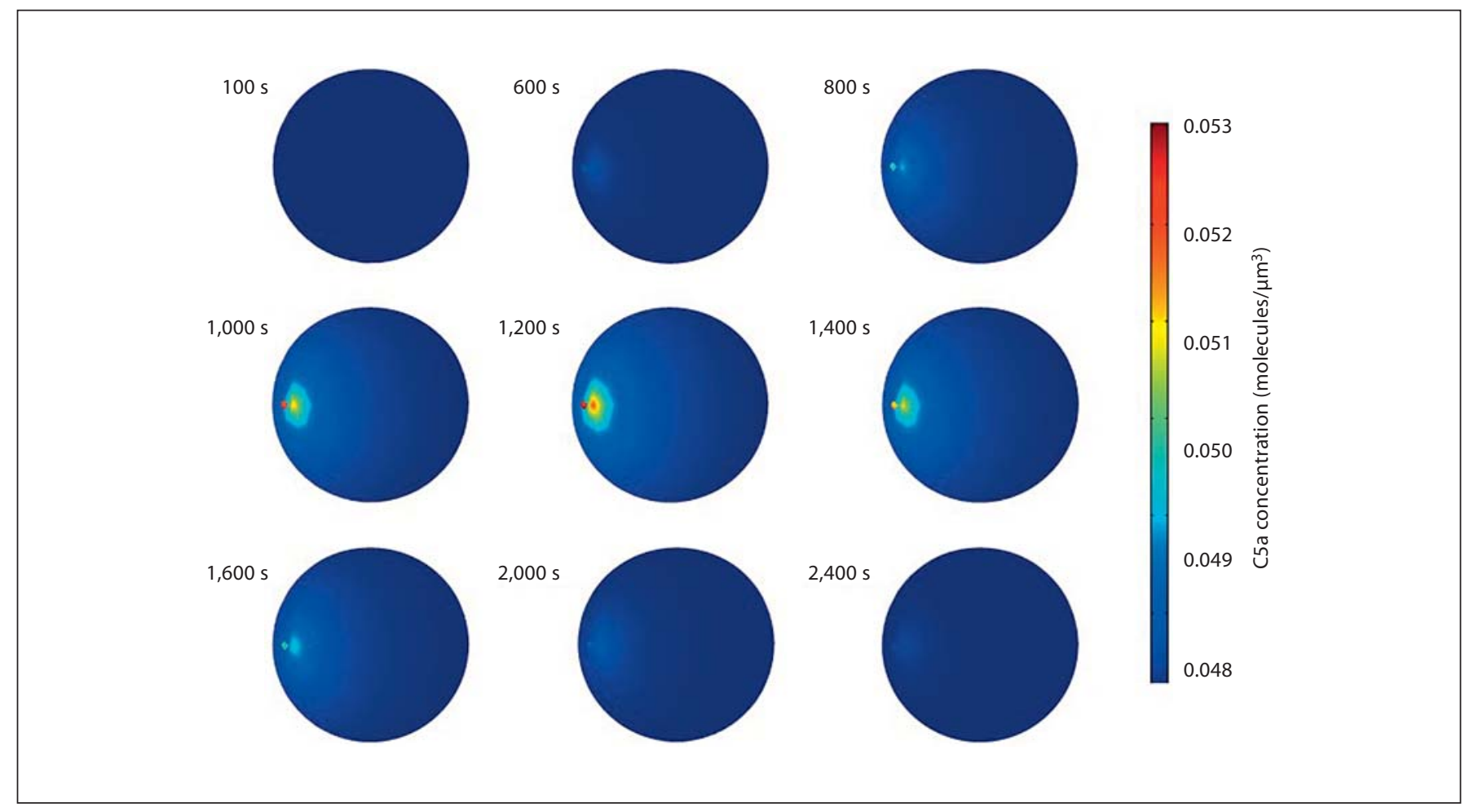

Fig. 5. Time course of the C5a plume near a neutrophil. Using the geometry in figure $1 \mathrm{~b}$, positioning the bacterium $0.5 \mu \mathrm{m}$ away from the cell surface, and assuming no convective mixing, the C5a concentration experienced by a neutrophil in close proxim- ity to a single cell of S. epidermidis was modeled. As in the capillary model (fig. 4), the concentration at the neutrophil surface never exceeds 0.053 molecules $/ \mu \mathrm{m}^{3}$, less than $10 \%$ of the $\mathrm{k}_{\mathrm{D}}$ value for the human $\mathrm{C} 5 \mathrm{a}$ receptor. 


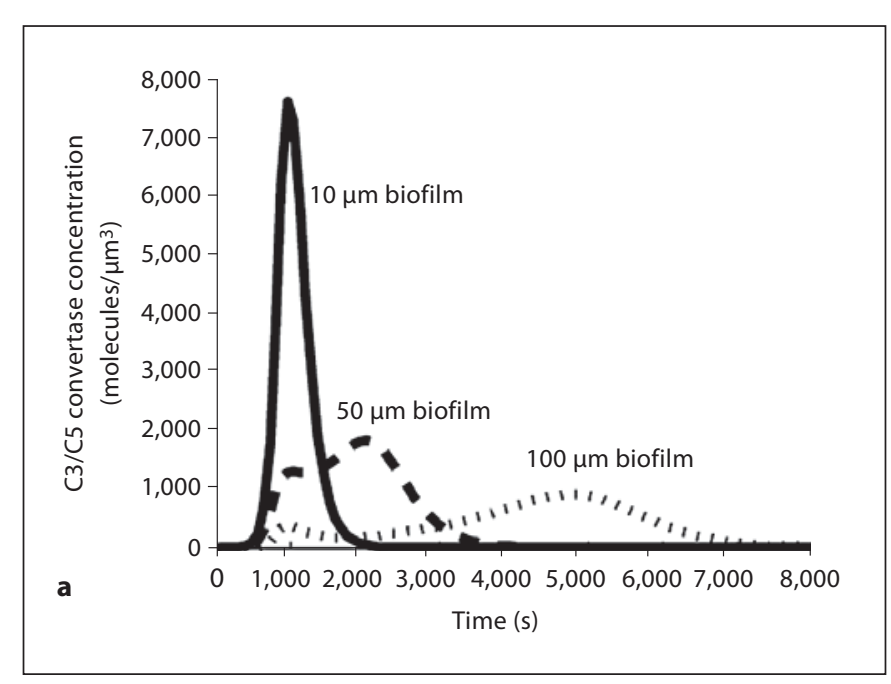

Fig. 6. Impact of biofilm magnitude EPS depth on C3/C5 convertase activity and $\mathrm{C} 5$ a production. a $\mathrm{C} 3 / \mathrm{C} 5$ convertase activity at the edge of a bacterium embedded in 10,50 , or $100 \mu \mathrm{m}$ of biofilm material. At EPS depths less than $10 \mu \mathrm{m}, \mathrm{C} 3$ diffusion remains rapid enough that no evidence of diffusion limitation is seen. However, at a thickness of $50 \mu \mathrm{m}$ of extracellular material, C3/C5 convertase activity is seen to be bimodal and prolonged. This effect is further amplified under $100 \mu \mathrm{m}$ of material, and is consistent with C3 limitation near the cell wall. b Similar plot showing the full spatiotemporal behavior under $100 \mu \mathrm{m}$ of biofilm. c Impact of biofilm thickness on C5a concentrations at the outer (plasma-facing) edge of a biofilm. Greater biofilm thickness produces high concentrations of $\mathrm{C} 5 \mathrm{a}$ for much longer periods of time than seen for a single cell (fig. 3).

\section{Discussion}

Here, we use a simplified quantitative model of C5a production upon plasma exposure to one of the most common etiologic agents of medical device infection and bacteremia, S. epidermidis. Our results indicate that the host interaction with this species when present as single cells or aggregates less than $10 \mu \mathrm{m}$ would be shortlived - roughly $15 \mathrm{~min}$ - and produce a local increase in C5a concentration an order of magnitude lower than the $\mathrm{k}_{\mathrm{D}}$ of the human C5a receptor. This type of interaction would be expected during a bacteremic episode where bacterial concentrations are typically less than 100 cells per milliliter of blood.

By extending the depth of the EPS to values that would be encountered in medical device-associated biofilms, the kinetics of opsonization and $\mathrm{C} 5 \mathrm{a}$ production become
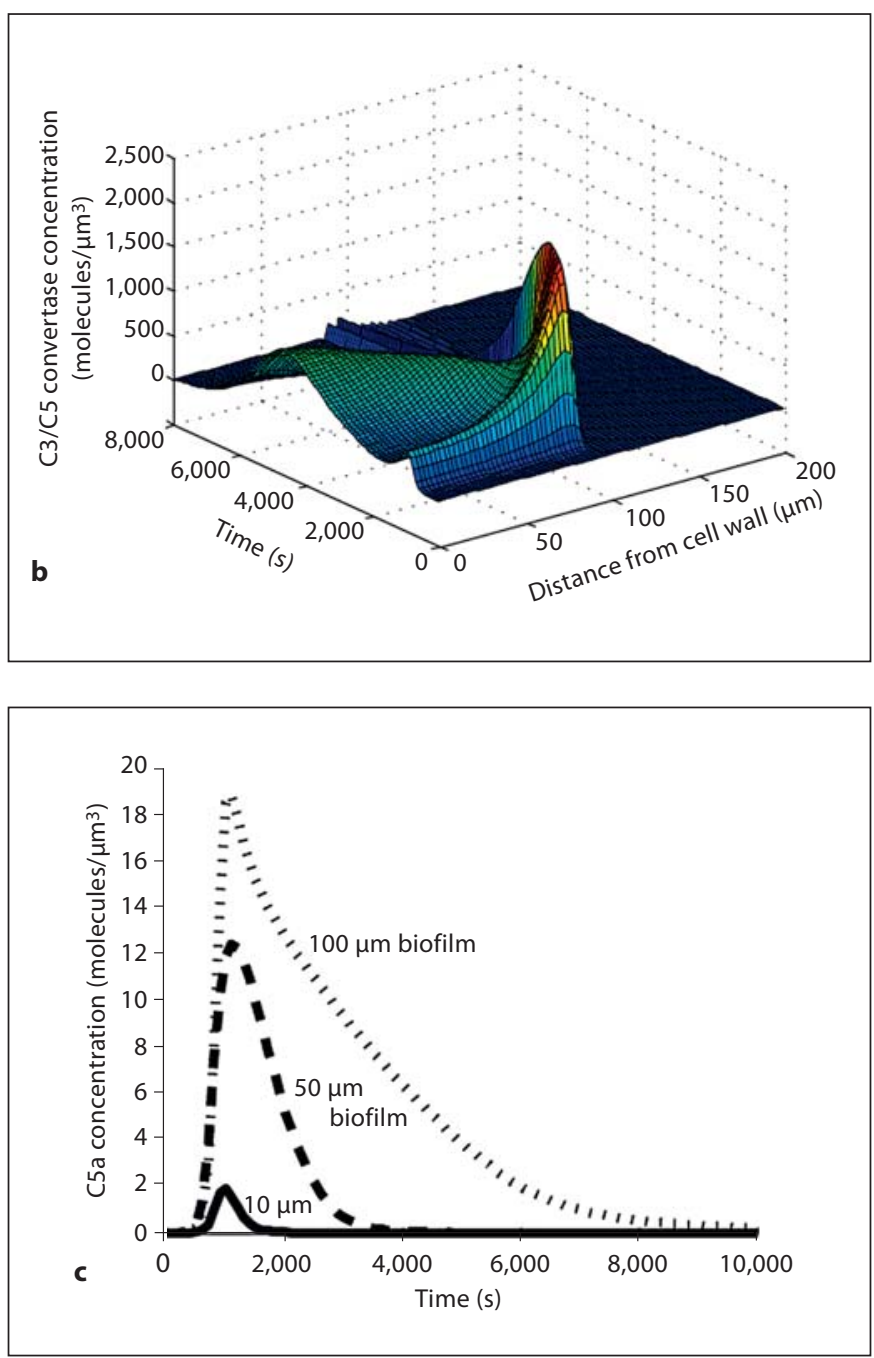

more complex. At thicknesses greater than $50 \mu \mathrm{m}$, we predict the time necessary for diffusion of mediators from the plasma into the biofilm will limit the rate of $\mathrm{C} 3$ / C5 convertase assembly and thus prolong the period of $\mathrm{C} 5$ a catalysis. Although our model considered only a static bacterial and biofilm architecture, it is interesting that if C3/C5 convertase activity in a 100- $\mu$ m-deep biofilm endures for more than $1 \mathrm{~h}$, bacterial growth and EPS secretion might allow for ongoing production of $\mathrm{C} 5 \mathrm{a}$ for an indefinite period of time.

Because this work is a computational model, it makes several simplifying assumptions and makes use of parameter values that do not fully apply to the biological situation described in the model. This restricts the extent to which we can expect the results of our model to agree with experimentally derived values. For example, to our knowledge, no data exist estimating the complement $\mathrm{C} 3$ 
binding site concentration for S. epidermidis EPS, and so we used data from a $C$. neoformans capsule as an approximation. Despite this limitation, our results for the timing and magnitude of $\mathrm{C} 5$ a production in plasma exposure to S. epidermidis biofilms agree with experimental data to within a factor of two, which suggests that the model captures the important components of the chemical and spatial dynamics.

Because our model is just a theoretical construct, it cannot be used to arrive at a precise estimate of true biochemical behavior. The parameters in our model have associated uncertainties which translate into model prediction uncertainty. A coarse estimate suggests uncertainties of about one order of magnitude. A more precise uncertainty quantification estimate is possible with sophisticated uncertainty quantification algorithms, but is beyond the scope of this article. However, the order-ofmagnitude agreement of our model with experiments allows us to make a series of directly testable hypotheses. First, our results indicate that the amount of C5a produced by single, or possibly a few, organisms in direct contact with an endothelial layer under physiological flow conditions would be insufficient to activate that cell layer by $\mathrm{C} 5$ a alone. Similarly, S. epidermidis brought into 1:1 stoichiometry with isolated neutrophils in plasma should, by creating a C5a concentration orders of magnitude lower than the $\mathrm{k}_{\mathrm{D}}$ of the receptor, produce very little cellular response. These could presumably be tested in an experimental system where concurrent detection pathways, particularly the TLR-4 pathway, were functionally silenced such that $\mathrm{C} 5 \mathrm{a}$ signaling became the predominant detection method. Additionally, time course determinations of fluorescently labeled $\mathrm{C} 3$ penetrating into thick staphylococcal biofilms visualized via scanning laser confocal microscopy would be expected to show delayed appearance of $\mathrm{C} 3$ into the deepest layers of the film, and the timescale of this delay should be readily measured experimentally.

Lastly, we would note that our findings of only modest C5a production by $S$. epidermidis are consistent with clinical experience with chronically indwelling bloodstream catheters, where colonization with this organism occurs frequently in the absence of any obvious clinical illness. As our model did not consider anything specific to S. epidermidis beyond its EPS thickness, it is also interesting to extrapolate our findings to other conditions associated with host interactions with single cells in the bloodstream where an associated underlying infection is not present. A good example of this would be the transient bacteremia associated with tooth brushing, and dental and medical procedures. Documented bacteremia of a duration $>1 \mathrm{~h}$ is known to occur following a dental extraction in the absence of systemic symptoms [33]. Our results suggest, at least in terms of innate host defense related to complement activation, that the intensity of the response to single cells in the dilute medium of the bloodstream might be insufficient to activate receptors necessary for cellular recognition of the event. This raises the possibility that the sensitivity of $\mathrm{C} 5$ a detection by various cell lines has been in part optimized to prevent substantial host responses to essentially nuisance bacterial exposures.

\section{Acknowledgments}

This work was supported by grants GM-069438 and GM081702 (J.G.Y. and D.M.B.), an NIH (5T35HL007690-30) summer scholar from University of Michigan Medical School, and a Medical Student Research Grant from the Society for Academic Emergency Medicine and Emergency Medicine Foundation (E.C.C.), and an Individual Grant from the Undergraduate Research Opportunities Program at the University of Colorado-Boulder (Y.Y.H.).

\section{References}

$>1$ Ehrnthaller C, Ignatius A, Gebhard F, Huber-Lang M: New insights of an old defense system: structure, function, and clinical relevance of the complement system. Mol Med 2011;17:317-329.

-2 Laarman A, Milder F, van Strijp J, Rooijakkers S: Complement inhibition by gram-positive pathogens: molecular mechanisms and therapeutic implications. J Mol Med 2010;88: 115-120.

3 Valencia G, Sarma JV, Younger JG: Complement C5 and early oxygen kinetics during murine sepsis. Acad Emerg Med 2005;12: 275-281.

$\checkmark 4$ Younger JG, Sasaki N, Delgado J, Ko AC, Nghiem TX, Waite MD, Till GO, Ward PA: Systemic and lung physiological changes in rats after intravascular activation of complement. J Appl Physiol 2001;90:2289-2295.

$\checkmark 5$ Younger JG, Sasaki N, Waite MD, Murray HN, Saleh EF, Ravage ZA, Hirschl RB, Ward PA, Till GO: Detrimental effects of complement activation in hemorrhagic shock. J Appl Physiol 2001;90:441-446.
6 Younger JG, Shankar-Sinha S, Mickiewicz M, Brinkman AS, Valencia GA, Sarma JV, Younkin EM, Standiford TJ, Zetoune FS, Ward PA: Murine complement interactions with Pseudomonas aeruginosa and their consequences during pneumonia. Am J Respir Cell Mol Biol 2003;29:432-438.

$>7$ Huber-Lang M, Younkin EM, Sarma JV, Riedemann N, McGuire SR, Lu KT, Kunkel R, Younger JG, Zetoune FS, Ward PA: Generation of C5a by phagocytic cells. Am J Pathol 2002;161:1849-1859. 
8 Czermak B, Sarma JV, Pierson C, Warner R, Huber-Lang M, Bless N, Scham H, Ward PA: Protective effects of C5a blockade in sepsis. Nat Med 1999;5:788-792.

-9 Huber-Lang M, Sarma JV, Lu KT, McGuire SR, Padgaonkar V, Guo RF, Younkin EM, Kunkel R, Ding JL, Erickson R, Curnutte J, Ward PA: Role of C5a in multiorgan failure during sepsis. J Immunol 2001;166:11931199.

10 Rittirsch D, Flierl M, Andeau B, Day D, Huber-Lang M, Mackay C, Zetoune FS, Gerard N, Cianlfone K, Kohl J, Gerard C, Sarma JV, Ward PA: Functional roles for C5a receptors in sepsis. Nat Med 2008; 14:551-557.

-11 Younger J, Bracho D, Chung-Esaki H, Lee M, Rana G, Sen A, Smith B, Jones A: Complement activation in emergency department patients with severe sepsis. Acad Emerg Med 2010;17:353-359.

-12 Pangburn M, Schreiber R, Muller-Eberhard $\mathrm{H}$ : Formation of the initial C3 convertase of the alternative complement pathway. Acquisition of C3b-like activities by spontaneous hydrolysis of the putative thioester in native C3. J Exp Med 1981;154:856-867.

13 Pangburn MK, Muller-Eberhard HJ: Initiation of the alternative complement pathway due to spontaneous hydrolysis of the thioester of C3a. Ann NY Acad Sci 1983;421:291298.

14 Pangburn MK, Schreiber RD, Muller-Eberhard HJ: Deficiency of an erythrocyte membrane protein with complement regulatory activity in paroxysmal nocturnal hemoglobinuria. Proc Natl Acad Sci USA 1983;80: 5430-5434.

15 Head M, Meryhew N, Runquist O: Mechanism and computer simulation of immune complex formation, opsonization, and clearance. J Lab Clin Med 1996;128:61-74.

16 Kalita J, Chandrashekar K, Hans R, Selvam $\mathrm{P}$, Newell MK: Computational modeling and simulation of the immune system. Int J Bioinform Res Appl 2006;2:63-68.
Korotaevskiy AA, Hanin LG, Khanin MA: Non-linear dynamics of the complement system activation. Math Biosci 2009;222: 127-143.

8 Liu B, Zhang J, Tan PY, Hsu D, Blom AM, Leong B, Sethi S, Ho B, Ding JL, Thiagarajan P: A computational and experimental study of the regulatory mechanisms of the complement system. PLoS Comput Biol 2011;7:1-16.

19 Nypaver CM, Thornton MM, Yin SM, Bracho DO, Nelson PW, Jones AE, Bortz DM, Younger JG: Dynamics of human complement-mediated killing of Klebsiella pneumoniae. Am J Respir Cell Mol Biol 2010;43: 585-590.

20 Otto M: Staphylococcus epidermidis' - the 'accidental' pathogen. Nat Rev Microbiol 2009;7:555-567.

21 Rogers KL, Fey PD, Rupp ME: Coagulasenegative staphylococcal infections. Infect Dis Clin North Am 2009;23:73-98.

22 Kristian SA, Birkenstock TA, Sauder U, Mack D, Gotz F, Landmann R: Biofilm formation induces C3a release and protects Staphylococcus epidermidis from IgG and complement deposition and from neutrophil-dependent killing. J Infect Dis 2008; 197:1028-1035.

23 Dzul S, Thornton MM, Hohne D, Stewart E, Shah A, Bortz D, Solomon MJ, Younger J: Contribution of the Klebsiella pneumoniae capsule to bacteria aggregate and biofilm microstructures. Appl Environ Microbiol 2011; 77:1777-1782.

24 Stewart E, Satorius A, Younger J, Solomon M: Impact of osmotic stress and sub-lethal antibiotic concentration on Staphylococcus epidermidis clustering and intercellular spacing in surface-adherent biofilms, in review.

25 Waharte F, Steenkeste K, Briandet R, Fontaine-Aupart MP: Diffusion measurements inside biofilms by image-based fluorescence recovery after photobleaching (FRAP) analysis with a commercial confocal laser scanning microscope. Appl Environ Microbiol 2010;76:5860-5869.
26 Kozel T, Wilson M, Welch W: Kinetic analysis of the amplification phase for activation and binding of $\mathrm{C} 3$ to encapsulated and nonencapsulated Cryptococcus neoformans. Infect Immun 1992;60:3122-3127.

27 Kozel TR, Tabuni A, Young BJ, Levitz SM: Influence of opsonization conditions on $\mathrm{C} 3$ deposition and phagocyte binding of largeand small-capsule Cryptococcus neoformans cells. Infect Immun 1996;64:2336-2338.

28 Satorius AE, Szafranski J, Rivera A, Ghanesan M, Solomon MJ, Newton DW, Bortz DM, Younger JG: Complement C5a generation by Staphylococcus epidermidis biofilms, submitted.

29 Stucker M, Baier V, Reuther T, Hoffman K, Kellam K, Altmeyer P: Capillary blood cell velocity in human skin capillaries located perpendicularly to the skin surface: measured by a new laser Doppler anemometer. Microvasc Res 1996;52:188-192.

30 Stocker T: Introduction to Climate Modelling. Berlin, Springer, 2011.

31 Murray JD: Mathematical Biology II: Spatial Models and Biomedical Applications, ed 3. New York, Springer, 2003.

-32 Gerard N, Gerard C: The chemotactic receptor for human C5a anaphylatoxin. Nature 1991;349:614-617.

33 Lockhart P, Brennan M, Sasser H, Fox P, Paster B, Bahrani-Mougeot F: Bacteremia associated with toothbrushing and dental extraction. Circulation 2008;117:3118-3125.

-34 Rawal N, Pangburn MK: Formation of highaffinity C5 convertases of the alternative pathway of complement. J Immunol 2001; 166:2635-2642.

35 Gressner OA, Koch A, Sanson E, Trautwein C, Tacke F: High C5a levels are associated with increased mortality in sepsis patients no enhancing effect by actin-free Gc-globulin. Clin Biochem 2008;41:974-980.

36 Pangburn M, Muller-Eberhard H: The C3 convertase of the alternative pathway of human complement. Enzymic properties of the bimolecular proteinase. Biochem J 1986;235: 723-730. 